\title{
Article \\ An Efficient Ground Moving Target Imaging Method for Airborne Circular Stripmap SAR
}

\author{
Yongkang Li ${ }^{1}{ }^{1 *}$, Tianyu Huo ${ }^{1}$, Chenxi Yang ${ }^{1}$, Tong Wang ${ }^{2}$, Juan Wang ${ }^{3}$ and Beiyu Li ${ }^{1}$ \\ 1 School of Electronics and Information, Northwestern Polytechnical University, Xi'an 710072, China; \\ hty1@mail.nwpu.edu.cn (T.H.); yangchengxi@mail.nwpu.edu.cn (C.Y.); byl2018@mail.nwpu.edu.cn (B.L.) \\ 2 National Laboratory of Radar Signal Processing, Xidian University, Xi'an 710071, China; \\ twang@mail.xidian.edu.cn \\ 3 China North Industries Group Corporation Limited, North Automatic Control Technology Institute, \\ Taiyuan 030006, China; jw03@mail.xidian.edu.cn \\ * Correspondence: ykli@nwpu.edu.cn
}

check for updates

Citation: Li, Y.; Huo, T.; Yang, C.; Wang, T.; Wang, J.; Li, B. An Efficient Ground Moving Target Imaging

Method for Airborne Circular

Stripmap SAR. Remote Sens. 2022, 14, 210. https://doi.org/10.3390/ rs14010210

Academic Editor: Hing Cheung So

Received: 28 October 2021

Accepted: 20 December 2021

Published: 4 January 2022

Publisher's Note: MDPI stays neutral with regard to jurisdictional claims in published maps and institutional affiliations.

Copyright: (C) 2021 by the authors. Licensee MDPI, Basel, Switzerland. This article is an open access article distributed under the terms and conditions of the Creative Commons Attribution (CC BY) license (https:// creativecommons.org/licenses/by/ $4.0 /)$.

\begin{abstract}
This paper studies the imaging of a ground moving target with airborne circular stripmap synthetic aperture radar (CSSAR). First, the range equation of a target moving with accelerations is developed. Then, a new range model of high accuracy is proposed, since the commonly used secondorder Taylor-approximated range model is inaccurate when the azimuth resolution is relatively high or the target moves with accelerations. The proposed range model also makes it easy to derive an accurate analytical expression for the target's 2-D spectrum. Third, based on the proposed range model, the target's 2-D spectrum is derived and an efficient imaging method is proposed. The proposed imaging method implements focusing via a phase multiplication in the 2-D frequency domain and utilizes the genetic algorithm to accomplish an efficient search of the parameters of the proposed range model. Finally, numerical experiments are conducted to validate the proposed range model and the proposed imaging method.
\end{abstract}

Keywords: circular stripmap synthetic aperture radar; imaging method; range equation model; ground moving target imaging; generic algorithm

\section{Introduction}

\subsection{Background of Airborne CSSAR and Ground Moving Target Imaging}

Airborne synthetic aperture radar (SAR) has been widely applied in both civilian and military fields due to its high-resolution observation capability and the ability to work regardless of weather and daylight [1-5]. The ground moving target imaging is a typical application of airborne SAR, which is of great value in air-to-ground surveillance and reconnaissance [6-11].

Airborne circular stripmap SAR (CSSAR) is a kind of SAR that travels along a horizonal circle with its antenna pointing to the outside of the horizonal circle [12-15], as shown in Figure 1. Benefiting from the circular path and the outward-pointing antenna, airborne CSSAR possess the advantages of a short revisit time and large coverage, and thus is an attractive tool for air-to-ground wide-area reconnaissance and time-sensitive targets surveillance [15].

SAR time-sensitive targets surveillance usually involves ground moving target imaging, and it has been a hot research topic in recent years. A key parameter in SAR imaging is the range equation of the target (i.e., the instantaneous range between the radar and the target), which determines the azimuth phase characteristics and the range cell migration (RCM) of the target [16]. The second-order Taylor approximated range equation model is a commonly used range model in SAR ground moving target imaging [9,10,17-21] It enables the derivation of an accurate analytical two-dimensional (2-D) spectrum of the target and thus can simplify the design of imaging method. However, its accuracy will 
be insufficient when the azimuth resolution is relatively high or the target moves with accelerations [22-24]. Although the higher order Taylor approximated range equation has higher accuracy, the derivation of the accurate 2-D spectrum is challenging and thus would complicate the design of imaging methods [25]. To address this problem, in [26-28], an equivalent hyperbola range model was proposed. This range model is much more precise than the second-order Taylor approximated range model. Besides, based on this range model, the analytical expression of the target's 2-D spectrum can be easily derived. However, it is only applicable to the constant-moving target in the case of straight path SAR.

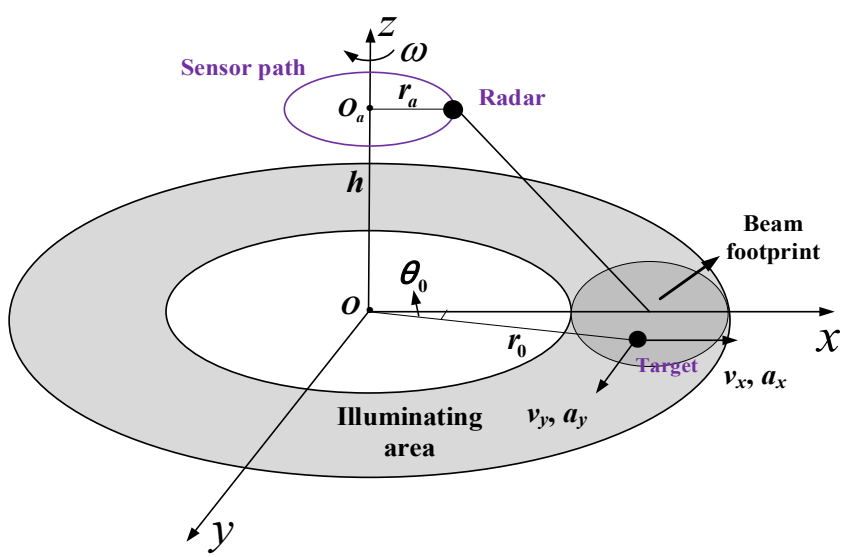

(a)

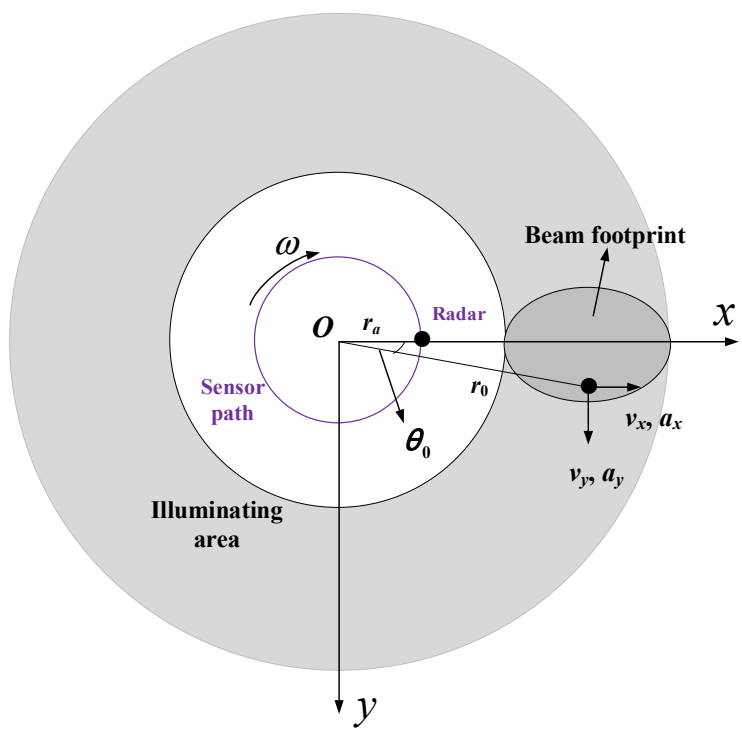

(b)

Figure 1. Geometry of airborne CSSAR. (a) 3-D view and (b) top view.

Due to the unknown motion of the target, SAR ground moving target imaging usually involves the searching of the target's motion parameters or other equivalent parameters (e.g., the Doppler parameters), the coefficients of the target's range equation, and the coefficients of the target's phase history [18-21,29-31]. For instance, for the imaging method proposed in [29], the two velocity parameters and two acceleration parameters of the target need to be searched. As for the imaging method proposed in [30], the coefficients of the phase history's second-order and third-order terms also require to be searched. These searches result in a huge computational load since the inefficient ergodic search method 
is usually used. Several papers proposed to utilize the second-order keystone transform and time-frequency techniques to replace the searching of the target's parameters [22-24]. However, the interpolation required by the second-order keystone transform and the searching involved in the time-frequency techniques make these methods still suffer from the problem of large computation load.

\subsection{Objectives of this Paper}

The main objective of this paper is to propose an imaging method that is able to efficiently focus a ground moving target with high quality, for airborne CSSAR with relatively high azimuth resolution. Based on the research gaps discussed in Section 1.1, studies are carried out to achieve the above objective from the following two aspects: (1) an accurate range equation model is proposed for airborne CSSAR ground moving target imaging; and (2) an efficient parameter searching strategy is proposed.

In [25], an advanced hyperbolic range model was proposed for medium-Earth-orbit SAR stationary scene imaging. This range model is much more precise than the secondorder Taylor-approximated range model. Inspired by this work, in this paper, a new range equation model is proposed for airborne CSSAR ground moving target imaging. The proposed range model has two main advantages: (1) its accuracy is higher than that of the second-order and third-order Taylor approximated range models; and (2) based on the proposed range model, an accurate analytical expression for the target's 2-D spectrum can be easily derived.

After deriving the target's 2-D spectrum based on the proposed range model, an efficient imaging method is proposed in this paper. The proposed imaging method implements focusing process efficiently via a phase multiplication in the 2-D frequency domain. Moreover, to accomplish an efficient searching of the parameters of the proposed range model, the genetic algorithm (GA) is utilized. GA is a powerful parallel stochastic algorithm that searches the optimal solution based on the principles of natural selection and natural genetics [32-37]. It is an efficient and robust optimization algorithm that can locate the global optimum in a multimodal landscape, and thus is utilized to reduce the computation load of the parameter searching involved in ground moving target imaging.

The rest of this paper is organized as follows. The airborne CSSAR geometry and the proposed range model are presented in Section 2. In Section 3, the target's 2-D spectrum based on the proposed range model is derived. In Section 4, the proposed imaging method is described in detail. Numerical experiments are conducted in Section 5 to validate the proposed range model and the proposed imaging method. Finally, discussions and conclusions are made in Sections 6 and 7, respectively.

\section{Proposed Range Model for CSSAR}

\subsection{Geometry}

The geometric relationship between an airborne CSSAR and a ground moving target is shown in Figure 1. The aircraft travels along a horizonal circle with height $h$ and radius $r_{a}$ at a constant angular velocity $\omega$, with its antenna pointing perpendicular to the aircraft direction fixedly. At the azimuth slow time $t_{a}=0$, the radar and the target are assumed to be located at $\left(r_{a}, 0, h\right)$ and $\left(r_{0} \cos \theta_{0}, r_{0} \sin \theta_{0}, 0\right)$, respectively, where $r_{0}$ is the distance between the target and the coordinate origin, and $\theta_{0}$ is the target's azimuth angle. In addition, it is assumed that the target moves with velocities $\left(v_{x}, v_{y}\right)$ and constant accelerations $\left(a_{x}, a_{y}\right)$ along the $\mathrm{x}$-and $\mathrm{y}$-axes.

According to the geometric relationship shown in Figure 1, the instantaneous distance from the radar to the target (i.e., the target's range equation) can be expressed as

$$
R\left(t_{a}\right)=\sqrt{\left(r_{0} \cos \theta_{0}+v_{x} t_{a}+\frac{1}{2} a_{x} t_{a}{ }^{2}-r_{a} \cos \left(\omega t_{a}\right)\right)^{2}+\left(r_{0} \sin \theta_{0}+v_{y} t_{a}+\frac{1}{2} a_{y} t_{a}{ }^{2}-r_{a} \sin \left(\omega t_{a}\right)\right)^{2}+h^{2}}
$$


Applying Taylor series expansion to (1) around the target's beam center crossing time, one can obtain

$$
R\left(t_{a}\right)=R_{c}+l_{1}\left(t_{a}-t_{a c}\right)+l_{2}\left(t_{a}-t_{a c}\right)^{2}++l_{3}\left(t_{a}-t_{a c}\right)^{3}+\cdots
$$

With

$$
\begin{gathered}
l_{1}=\frac{v_{t c}\left(r_{c}-r_{a}\right)}{R_{c}} \\
l_{2}=\frac{v_{t a}^{2}-2 r_{a} \omega \cdot v_{t a}+r_{a} r_{c} \omega^{2}}{2 R_{c}}+\frac{a_{t c}\left(r_{c}-r_{a}\right)}{2 R_{c}}+\frac{h^{2} v_{t c}^{2}}{2 R_{c}^{3}}
\end{gathered}
$$

$$
+\frac{v_{t a}^{3}\left(r_{c}-r_{a}\right)^{3}}{2 R_{c}^{5}}
$$

$$
\begin{aligned}
& v_{t c}=v_{x} \cos \theta_{c}+v_{y} \sin \theta_{c} \\
& v_{t a}=v_{y} \cos \theta_{c}-v_{x} \sin \theta_{c} \\
& a_{t c}=a_{x} \cos \theta_{c}+a_{y} \sin \theta_{c} \\
& a_{t a}=a_{y} \cos \theta_{c}-a_{x} \sin \theta_{c}
\end{aligned}
$$

where $t_{a c}$ is the beam center crossing time, $R_{c}$ is the range between the radar and the target at $t_{a}=t_{a c}, r_{c}$ is the range between the origin of coordinate and the target at $t_{a}=t_{a c}, v_{t c}$ and $a_{t c}$ are the projections of the target's velocity and acceleration onto the vertical direction of the radar platform velocity at $t_{a}=t_{a c}$, respectively, and $v_{t a}$ and $a_{t a}$ are the projections of the target's velocity and acceleration onto the direction of the radar platform velocity at $t_{a}=t_{a c}$, respectively, and $\theta_{c}$ is the target's azimuth angle at $t_{a}=t_{a c}$.

The accuracy of the Taylor-approximated range equation increases with the order of Taylor series expansion. However, when this order is more than two, the derivation of an accurate analytical 2-D spectrum and the design of imaging method will be challenging. Thus, a second-order Taylor expansion is usually applied to the target's range equation. Nevertheless, when the azimuth resolution goes high or the target moves with accelerations, the accuracy of the second-order Taylor-approximated range equation would be insufficient. Herein, a novel range equation model is proposed. The accuracy of the proposed range model is even higher than that of the third-order Taylor approximated range equation. Moreover, based on the proposed range model, an accurate analytical expression for the target's 2-D spectrum can be easily derived.

\subsection{Proposed Range Model}

In order to accomplish an accurate imaging of the target and to allow for an accurate analytical 2-D spectrum to benefit the design of imaging method, the following range equation model is proposed

$$
R_{p}\left(t_{a}\right)=\sqrt{R_{c}^{2}+v_{e}^{2} t_{a}^{2}+\alpha t_{a}}+\beta t_{a}
$$

As can be seen from (10), there are three variables (i.e., $v_{e}, \alpha$, and $\beta$ ). In order to obtain the expression for these parameters, a third-order Taylor series expansion is applied to (10) and then we obtain

$$
R_{p}\left(t_{a}\right) \approx R_{c}+\left(\beta+\frac{\alpha}{2 R_{c}}\right)\left(t_{a}-t_{a c}\right)+\left(\frac{v_{e}^{2}}{2 R_{c}}-\frac{\alpha^{2}}{8 R_{c}^{3}}\right)\left(t_{a}-t_{a c}\right)^{2}+\left(-\frac{\alpha v_{e}^{2}}{4 R_{c}^{3}}+\frac{\alpha^{3}}{16 R_{c}^{5}}\right)\left(t_{a}-t_{a c}\right)^{3}
$$


Letting the coefficients of the first, second, and third terms of (11) be equal to those of (2), respectively, one can obtain the expressions for $v_{e}, \alpha$, and $\beta$ as follows

$$
\begin{gathered}
v_{e}=\sqrt{\left(\frac{R_{c} l_{3}}{l_{2}}\right)^{2}+2 R_{c} l_{2}} \\
\alpha=-\frac{2 R_{c}^{2} l_{3}}{l_{2}} \\
\beta=l_{1}+\frac{R_{c} l_{3}}{l_{2}}
\end{gathered}
$$

From (10) it can be seen that the proposed range model consists of a hyperbola and a linear term. The proposed range model has two advantages: (1) it allows for an accurate analytical 2-D spectrum which will benefit the design of imaging algorithm (the target's 2-D spectrum will be presented in the next section); and (2) its accuracy is significantly higher than those of the commonly used second-order and third-order Taylor-approximated range equations.

In the following, the accuracy superiority of the proposed range model over the second-order and third-order Taylor approximated range models are demonstrated via numerical experiments.

The phase error is utilized to indicate the accuracies of the proposed range equation model as well as the second-order and third-order Taylor-approximated range equations, and it is calculated as follows

$$
\Omega=\max _{t_{a} \in\left[-T_{a} / 2, T_{a} / 2\right]}\left\{4 \pi \cdot \frac{\left|R\left(t_{a}\right)-R^{\prime}\left(t_{a}\right)\right|}{\lambda}\right\}
$$

where $R\left(t_{a}\right)$ is the target's actual range equation, $R^{\prime}\left(t_{a}\right)$ indicates the approximated range models (i.e., the proposed range model, the second-order and third-order Taylor approximated range models), $\lambda$ is the wavelength. $T_{a}$ is the synthetic aperture time, and it can be approximately expressed as [16]

$$
\begin{aligned}
T_{a} & \approx \frac{\theta_{b w} R_{c}}{v_{g}} \\
& \approx 0.886 \cdot \frac{\lambda R_{c}}{2 v_{a} \rho_{a}}
\end{aligned}
$$

where $\theta_{b w}$ is the $3 \mathrm{~dB}$ beamwidth of the radar, $\rho_{a} \approx 0.886 \lambda v_{g} /\left(2 \theta_{b w} v_{a}\right)$ is the azimuth resolution, $v_{g}=\omega \cdot r_{c}$ is the velocity of the beam footprint along the ground, and $v_{a}=\omega \cdot r_{a}$ is the velocity of the radar platform.

In Figures 2 and 3, the phase errors under different velocities and accelerations are illustrated, respectively. The color indicates the value of the phase error with the unit being radian. Comparing Figure $2 a$ with Figure $2 b$ and Figure $3 a$ with Figure $3 b$, it can be seen that the phase errors of the proposed range model are much smaller than that of the second-order Taylor approximated range equation. Moreover, by comparing Figure $2 \mathrm{a}$ with Figure $2 \mathrm{c}$ and Figure 3a with Figure 3c, one can see that the phase errors of the proposed range equation model are even smaller than that of the third-order Taylor approximated range equation. These results validate the accuracy superiority of the proposed range equation model over the second-order and third-order Taylor approximated range equations. The parameters of the CSSAR for the simulations are presented in Table 1. 


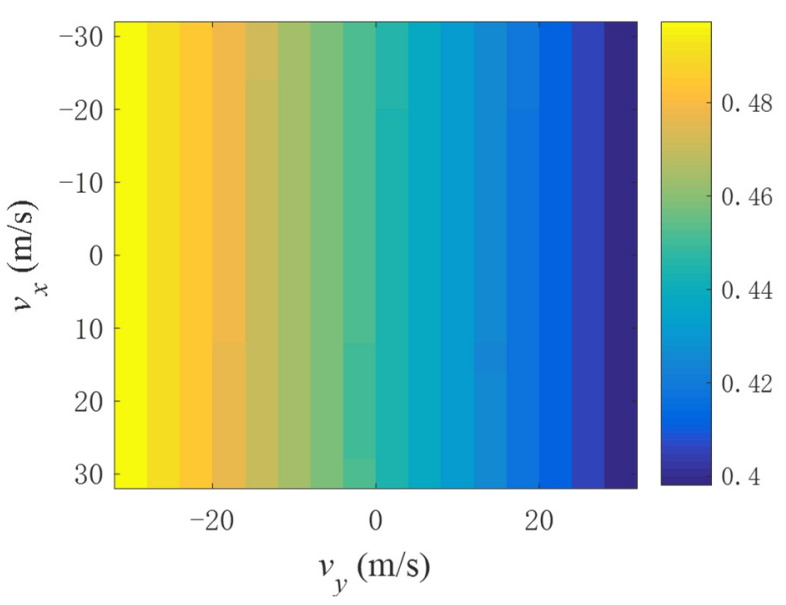

(a)

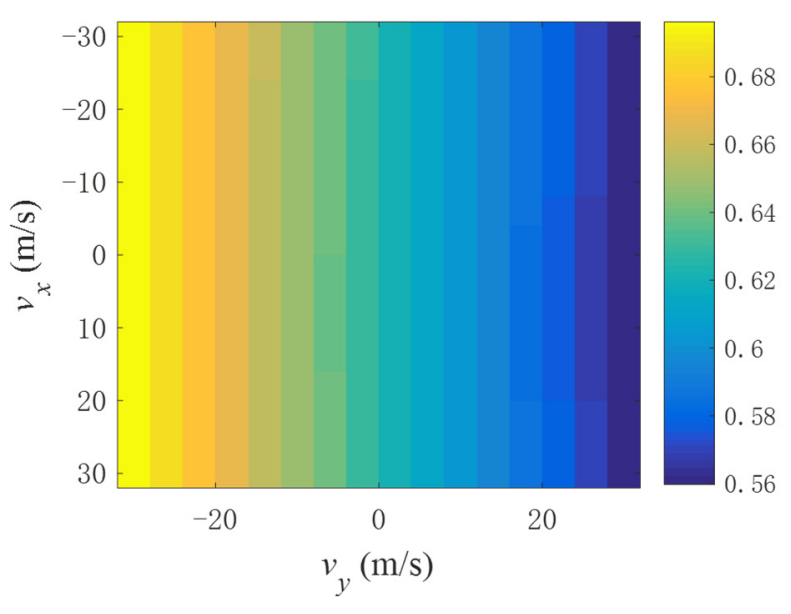

(c)

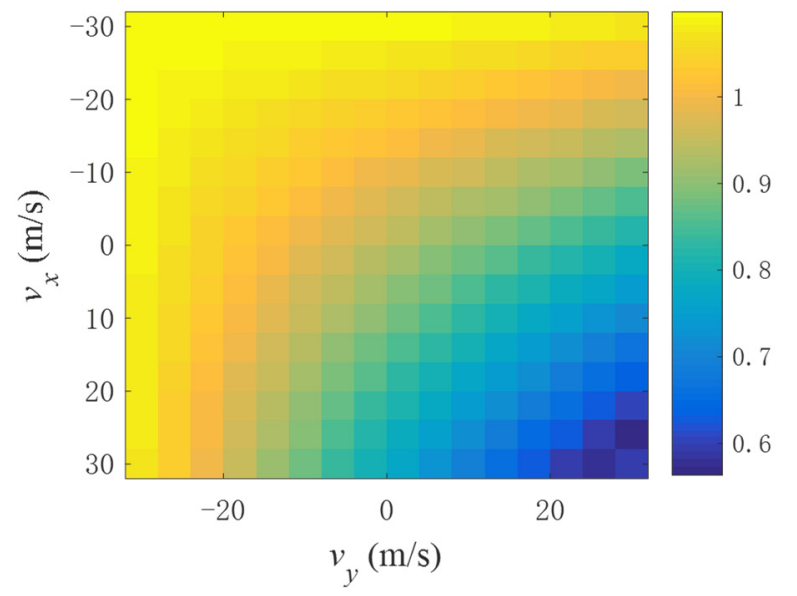

(b)

Figure 2. Phase errors under different velocities. (a) Proposed range equation, (b) second-order Taylor approximated range equation, and (c) third-order Taylor approximated range equation. The parameters of the target are: $R_{c}=20 \mathrm{~km}, a_{x}=0.3 \mathrm{~m} / \mathrm{s}^{2}$, and $a_{y}=0.2 \mathrm{~m} / \mathrm{s}^{2}$.

Table 1. Parameters of airborne CSSAR for simulation.

\begin{tabular}{cc}
\hline Parameter & Value \\
\hline Carrier frequency & $10 \mathrm{GHz}$ \\
\hline Range bandwidth & $150 \mathrm{MHz}$ \\
\hline Sampling frequency & $180 \mathrm{MHz}$ \\
\hline Pulse repetition frequency & $1500 \mathrm{~Hz}$ \\
\hline Azimuth resolution & $1 \mathrm{~m}$ \\
\hline Illumination time & $1.69 \mathrm{~s}$ \\
\hline Flying radius & $2.3 \mathrm{~km}$ \\
\hline Platform altitude & $8 \mathrm{~km}$ \\
\hline Platform velocity & $125 \mathrm{~m} / \mathrm{s}$ \\
\hline Ground range of scene center & $16 \mathrm{~km}$ \\
\hline
\end{tabular}




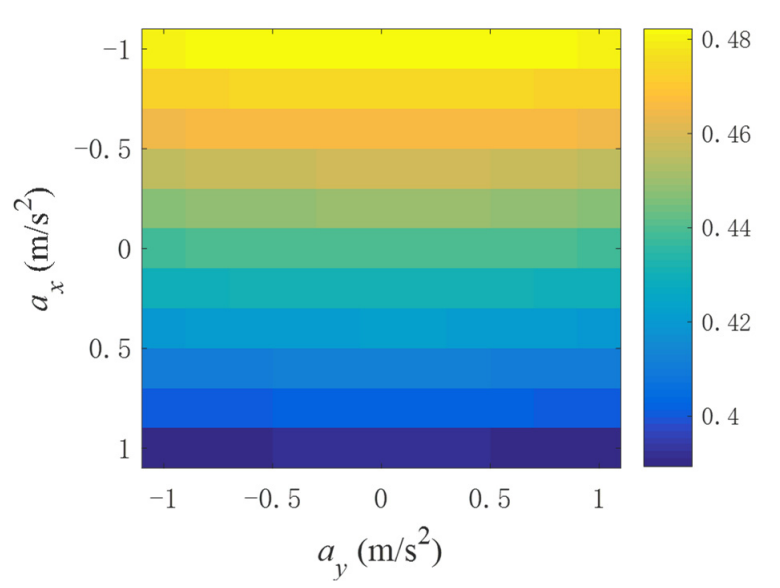

(a)

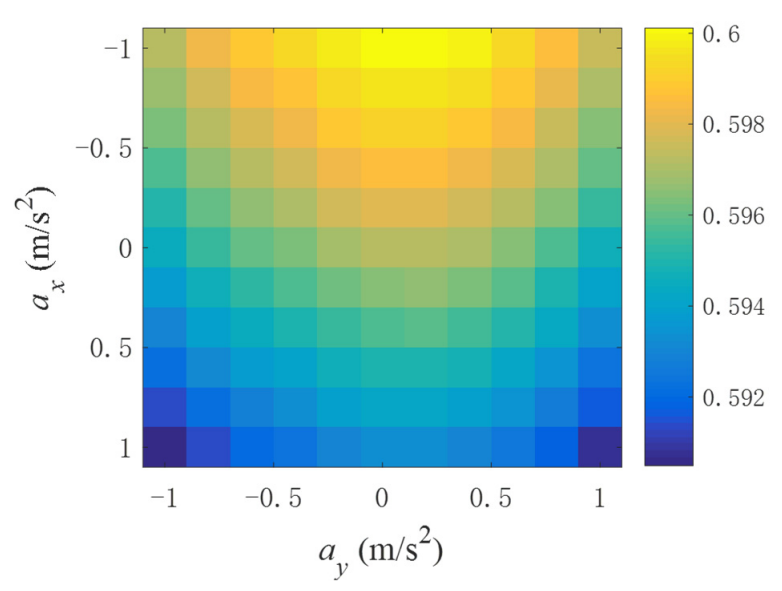

(c)

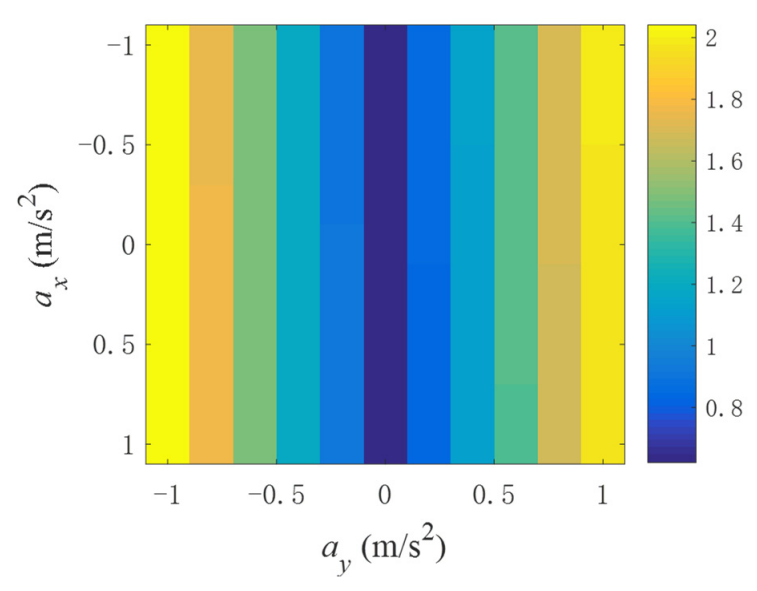

(b)

Figure 3. Phase errors under different accelerations. (a) Proposed range equation, (b) secondorder Taylor approximated range equation, and (c) third-order Taylor approximated range equation. The parameters of the target are: $R_{c}=20 \mathrm{~km}, v_{x}=5 \mathrm{~m} / \mathrm{s}$, and $v_{y}=13 \mathrm{~m} / \mathrm{s}$.

\section{Target's 2-D Spectrum Based on the Proposed Range Model}

After demodulating to the baseband, the target's signal in the raw data domain can be expressed as

$$
s\left(t_{r}, t_{a}\right)=w_{r}\left(t_{r}-\frac{2 R_{p}\left(t_{a}\right)}{c}\right) \exp \left\{j \pi k_{r}\left[t_{r}-\frac{2 R_{p}\left(t_{a}\right)}{c}\right]^{2}\right\} \times w_{a}\left(t_{a}\right) \exp \left\{-j \pi \frac{4 R_{p}\left(t_{a}\right)}{\lambda}\right\}
$$

where $w_{r}(\cdot)$ and $w_{a}(\cdot)$ denote the range and azimuth envelopes, respectively, $t_{r}$ is the range time, $k_{r}$ is the chirp rate of the transmitted signal, $c$ is the speed of light, and $f_{c}$ is the carrier frequency.

Applying a Fourier transform in range to (17), one can obtain the target's signal in the range frequency domain as follows

$$
S\left(f_{r}, t_{a}\right)=W_{r}\left(f_{r}\right) \exp \left\{-j \pi \frac{f_{r}^{2}}{k_{r}}\right\} \times w_{a}\left(t_{a}\right) \exp \left\{-j \pi \frac{4\left(f_{c}+f_{r}\right) R_{P}\left(t_{a}\right)}{c}\right\}
$$

where $f_{r}$ denotes the range frequency and $W_{r}(\cdot)$ denotes the range frequency envelope.

To obtain the target's 2-D spectrum, a Fourier transform in azimuth is applied to (18):

$$
S\left(f_{r}, f_{a}\right)=\int_{-\infty}^{+\infty} S\left(f_{r}, t_{a}\right) \exp \left\{-j 2 \pi f_{a} t_{a}\right\} d t_{a}
$$


where $f_{a}$ denotes the azimuth frequency. To obtain the analytical expression for the target's 2-D spectrum, the principle of stationary phase [16] is utilized. Based on (10), (18), and (19), the stationary phase point is given by

$$
t_{a}^{*}=-\frac{\sqrt{4 v_{e}^{2} R_{c}^{2}-\alpha^{2}}}{2 v_{e}^{2}}\left[\frac{\left(\frac{c f_{a}}{2 v_{e}\left(f_{c}+f_{r}\right)}+\frac{\beta}{v_{e}}\right)}{\sqrt{1-\left(\frac{c f_{a}}{2 v_{e}\left(f_{c}+f_{r}\right)}+\frac{\beta}{v_{e}}\right)^{2}}}\right]-\frac{\alpha}{2 v_{e}^{2}}
$$

According to the principle of stationary phase, the target's 2-D spectrum can be obtained as follows

$$
\begin{aligned}
S\left(f_{r}, f_{a}\right) & =S\left(f_{r}, t_{a}^{*}\right) \exp \left\{-j 2 \pi f_{a} t_{a}^{*}\right\} \\
& =W_{r}\left(f_{r}\right) W_{a}\left(f_{a}\right) \exp \left\{j \theta\left(f_{r}, f_{a}\right)\right\}
\end{aligned}
$$

With

$$
\begin{aligned}
\theta\left(f_{r}, f_{a}\right) & =-\frac{2 \pi \sqrt{4 v_{e}^{2} R_{c}^{2}-\alpha^{2}}}{\lambda v_{e}} \sqrt{1-\left(\frac{\lambda f_{a}}{2 v_{e}}+\frac{\beta}{v_{e}}\right)^{2}+\left(1-\frac{\beta}{v_{e}}\left(\frac{\lambda f_{a}}{2 v_{e}}+\frac{\beta}{v_{e}}\right)\right)\left(\frac{2 f_{r}}{f_{c}}\right)+\left(1-\left(\frac{\beta}{v_{e}}\right)^{2}\right)^{2}\left(\frac{f_{r}}{f_{c}}\right)^{2}} \\
& +\frac{2 \pi \alpha \beta\left(f_{c}+f_{r}\right)}{c v_{e}^{2}}+\frac{\pi \alpha f_{a}}{v_{e}^{2}}-\frac{\pi f_{r}{ }^{2}}{k_{r}}
\end{aligned}
$$

where $W_{a}(\cdot)$ denotes the azimuth frequency envelope.

From the above derivations it can be seen that the analytical expression of the target's 2-D spectrum can be easily derived without any approximations being made. Therefore, the proposed range model enables the derivation of an accurate analytical expression for the target's 2-D spectrum.

\section{Proposed Imaging Method}

In this section, based on the proposed range model and the derived 2-D spectrum of the target, a novel ground moving target imaging method is presented. First, the imaging process is modeled as an optimization problem. Then, the details on utilizing the GA to solve the optimization problem are described. Finally, the flowchart of the proposed imaging method is presented.

\subsection{Optimization Modeling}

From (21), it can be seen that the target focusing can be accomplished via compensating the phase modulation in the 2-D frequency domain and a 2-D inverse Fourier transform. Based on (21) and (22), the filter for compensating the phase modulation is constructed as follows:

$$
H\left(f_{r}, f_{a} ; v_{e}, \alpha, \beta\right)=\exp \left\{\frac{j \pi f_{r}^{2}}{k_{r}}+\frac{j 2 \pi \sqrt{4 v_{e}^{2} R_{c}^{2}-\alpha^{2}}}{\lambda v_{e}} \sqrt[\begin{array}{c}
1-\left(\frac{f_{a}}{2 \lambda v_{e}}+\frac{\beta}{v_{e}}\right)^{2}+\left[1-\frac{\beta}{v_{e}}\left(\frac{f_{a}}{2 \lambda v_{e}}+\frac{\beta}{v_{e}}\right)\right]\left(\frac{2 f_{r}}{f_{c}}\right) \\
+\left[1-\left(\frac{\beta}{v_{e}}\right)^{2}\right]\left(\frac{f_{r}}{f_{c}}\right)^{2}
\end{array}]{\begin{array}{l}
+[1 \\
f^{2}
\end{array}}\right\}
$$

From (23) it can be seen that this filter depends on the three variables of the proposed range model. In practice, these three variables are usually unknown. Therefore, in the process of target imaging, we need to search these variables, and the target will be completely focused when the searched values match the true values of these variables. Given that the contrast of the target's image will reach its maximum value when the target is completely focused, the process of imaging can be modeled as the following optimization problem

$$
\left(\hat{v}_{e}, \hat{\alpha}, \hat{\beta}\right)=\underset{\substack{v_{e, t} \in\left[v_{e, \min }, v_{e, \max }\right] \\ \alpha_{t} \in\left[\alpha_{\min }, \alpha_{\max }\right] \\ \beta_{t} \in\left[\beta_{\min }, \beta_{\max }\right]}}{\operatorname{argmax}}\left\{\operatorname{Contrast}\left[s\left(t_{r}, t_{a} ; v_{e, t}, \alpha_{t}, \beta_{t}\right)\right]\right\}
$$


With

$$
\begin{aligned}
\text { Contrast }\left[s\left(t_{r}, t_{a} ; v_{e, t}, \alpha_{t}, \beta_{t}\right)\right]= & \frac{\sqrt{E\left\{\left[\left|s\left(t_{r}, t_{a} ; v_{e, t}, \alpha_{t}, \beta_{t}\right)\right|^{2}-E\left(\left|s\left(t_{r}, t_{a} ; v_{e, t}, \alpha_{t}, \beta_{t}\right)\right|^{2}\right)\right]^{2}\right\}}}{E\left\{\left|s\left(t_{r}, t_{a} ; v_{e, t}, \alpha_{t}, \beta_{t}\right)\right|^{2}\right\}} \\
& s\left(t_{r}, t_{a} ; v_{e, t}, \alpha_{t}, \beta_{t}\right)=\operatorname{IDFT}_{2}\left\{S\left(f_{r}, f_{a}\right) \cdot H\left(f_{r}, f_{a} ; v_{e, t}, \alpha_{t}, \beta_{t}\right)\right\}
\end{aligned}
$$

where $\operatorname{IDFT}_{2}(\cdot)$ indicates the 2-D inverse Fourier transform, $E(\cdot)$ presents the spatial average, Contrast $(\cdot)$ is the image contrast, $\hat{v}_{e}, \hat{\alpha}$, and $\hat{\beta}$ are the estimates of the corresponding variables, $v_{e, \min }$ and $v_{e, \max }$ are the minimum and maximum values of $v_{e}$, respectively, $\alpha_{\min }$ and $\alpha_{\max }$ are the minimum and maximum values of $\alpha$, respectively, and $\beta_{\min }$ and $\beta_{\max }$ are the minimum and maximum values of $\beta$, respectively.

From the above analysis it can be seen that the solution for the optimization problem is the optimal estimation of the three parameters. Thus, the focused SAR image of the target can be given by

$$
I\left(t_{r}, t_{a}\right)=\operatorname{IDFT}_{2}\left\{S\left(f_{r}, f_{a}\right) \cdot H\left(f_{r}, f_{a} ; \hat{v}_{e}, \hat{\alpha}, \hat{\beta}\right)\right\}
$$

If the traditional ergodic search method is utilized to solve the above optimization problem, the computation load will be very high since it is a three-dimensional search. To decrease the computation load, in the following subsection, the GA is used to solve this optimization problem.

\subsection{Solving the Optimization Problem via GA}

GA starts with encoding the parameters of the search space in the form of a chromosomelike structure. Then, an initial population (a population is constituted by a group of chromosomes) is generated. After that, the fitness value of each individual chromosome of the population is calculated based on a defined fitness function. Subsequently, a selection operation is performed, in which the fittest chromosomes will be chosen with a larger probability. Then, the selected chromosomes are used as parents to produce the next generation by the crossover and mutation operations. This procedure is repeated until the stopping criterion is met.

In this subsection, GA is utilized to solve the optimization problem presented in (24). The specific steps are explained as follows.

(1) Step 1-Coding: Each individual chromosome of the population is encoded as a binary string with a fixed length. The length of the string depends on the range of the parameters to be searched and the required accuracy. For example, the length of the string for the parameter $v_{e}$ should be $2^{l_{v e}-1}<\left(v_{e, \max }-v_{e, \min }\right) / \Delta v_{e}<2^{l_{v e}}$, where $l_{v e}$ is the length of the string for $v_{e}$, and $\Delta v_{e}$ is the encoding accuracy for $v_{e}$.

(2) Step 2-Population initialization: The population size NP, the maximum number of generations $G_{\max }$, and the generation counter $g$ are initialized. All individuals of the population in the 0 th generation are generated randomly.

(3) Step 3-Calculating the fitness: Since the optimization problem is based on maximizing the contrast of the target's image, the fitness value of a chromosome is chosen to be the contrast of the image that is focused with the parameters corresponding to this chromosome. The image is obtained via (26), and the contrast is calculated via (25).

(4) Step 4-Selection: The selection operation keeps good chromosomes and eliminates inferior ones. In this paper, to improve the efficiency, the roulette wheel selection rule [33] is adopted, and the selection probability for each chromosome is calculated as follows:

$$
p\left(\mathrm{CP}_{g, n}\right)=\frac{f\left(\mathrm{CP}_{g, n}\right)}{\sum_{n=1}^{N} f\left(\mathrm{CP}_{g, n}\right)}
$$


where $f(\cdot)$ is the fitness function, and $\mathrm{CP}_{g, n}$ is the $n$th chromosome of the population in $g$ th generation. In addition, the optimal individual preservation strategy [32] is used. In other words, the optimal individual of the parent population will directly enter the offspring population.

(5) Step 5-Crossover and mutation: In this paper, the single-point crossover operator and simple mutation operator [33] are applied to the selected chromosomes to produce the population of the next generation.

(6) Step 6-Judgment: If $g$ is equal to $G_{\max }$ or the maximum fitness value remains stable, jump to step 7. Otherwise, return to step 3.

(7) Step 7-Output the optimal solution: The chromosome that has the largest fitness value is the optimal solution, and the corresponding parameters are the solutions for the optimization problem. To obtain the solutions for the optimization problem, a decoding operation should be performed with two steps (taking $v_{e}$ for example): (1) convert the binary string to the decimal number $v_{e}^{\prime}$; (2) calculate the actual value of $v_{e}$ by (29).

$$
\hat{v}_{e}=v_{e, \min }+v_{e}^{\prime} \cdot \frac{v_{e, \max }-v_{e, \min }}{2^{l_{v e}}-1}
$$

\subsection{Flowchart of the Proposed Imaging Method}

Based on the analyses presented in Sections 4.1 and 4.2, the flowchart of the proposed imaging method is presented in Figure 4. It starts with the target signal that has been extracted. First, a 2-D Fourier transform is applied to the target signal to transform the target signal into the 2-D frequency domain. Then, the strategy presented in Section 4.2 is utilized to solve the imaging optimization problem shown in (24). Note that, in using GA to solve the optimization problem, the fitness function is the contrast of the image that is focused with each chromosome, and the image is obtained via (26). Therefore, it is essential to use GA to find the chromosome that obtains the largest image contrast. Finally, with the optimal solution obtained by GA, the target focusing is performed efficiently via (27).

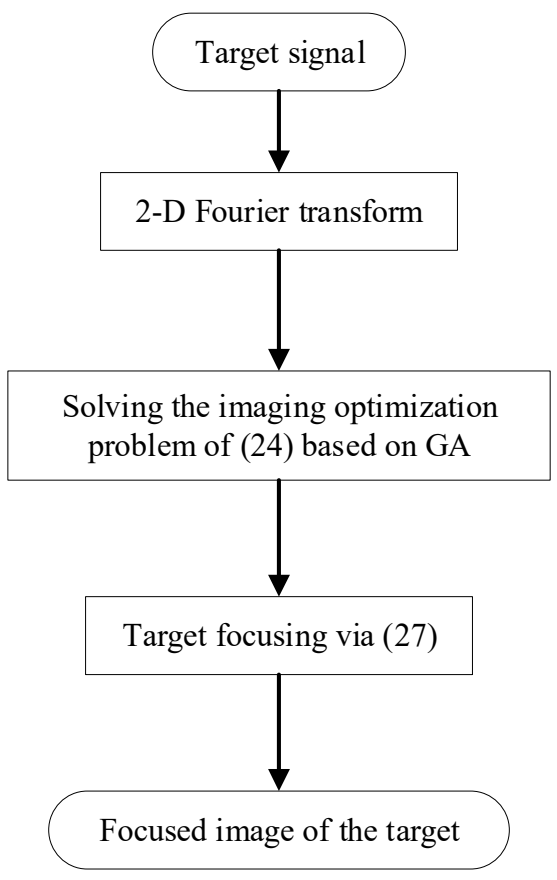

Figure 4. Flowchart of the proposed imaging method. 


\section{Experimental Results}

\subsection{Validation of the Proposed Range Model}

In Section 2.2, we have demonstrated the accuracy superiority of the proposed range model over the second-order and third-order Taylor approximated range models. In this subsection, to further validate the proposed range model, the focusing results based on the original range equation, the proposed range model, the second-order and third-order Taylor-approximated range models are analyzed and compared. The parameters of CSSAR used in the experiments are given in Table 1.

Figure 5 shows the focusing results, with the expanded range and azimuth profiles of the focused target being plotted. In addition, the focusing quality parameters are measured and the results are shown in Table 2 . The measured quality parameters include the azimuth impulse response width (IRW) broadening, the peak sidelobe ratio (PSLR), and the integrated sidelobe ratio (ISLR) [16]. From Figure 5 and Table 2 it can be seen that the focusing quality of these four range models in range are almost the same. This indicates that the accuracies of these range models are sufficient for range compression and RCM correction. However, it can also be seen that the azimuth profile obtained based on the second-order Taylor approximated range model suffers significant sidelobe asymmetry, IRW broadening, PSLR and ISLR losses. Besides, the focusing result obtained based on the third-order Taylor-approximated range model also suffers azimuth IRW broadening, PSLR and ISLR losses. Nevertheless, quality parameters obtained based on the proposed range model are very close to those obtained based on the original range equation, which indicates that the target is accurately focused.

Table 2. Measured quality parameters.

\begin{tabular}{ccccc}
\hline & & IRW Broadening & $\begin{array}{c}\text { PSLR } \\
(\mathbf{d B})\end{array}$ & $\begin{array}{c}\text { ISLR } \\
(\mathbf{d B})\end{array}$ \\
\hline \multirow{2}{*}{ Original range model } & Azimuth & $0.00 \%$ & -13.23 & -10.12 \\
\cline { 2 - 5 } & Range & $0.00 \%$ & -13.26 & -10.04 \\
\hline \multirow{2}{*}{ Proposed range model } & Azimuth & $0.00 \%$ & -13.12 & -9.86 \\
\cline { 2 - 5 } & Range & $0.00 \%$ & -13.26 & -10.04 \\
\hline $\begin{array}{c}\text { Second-order Taylor } \\
\text { approximated range model }\end{array}$ & Azimuth & $2.03 \%$ & -11.05 & -9.06 \\
\cline { 2 - 5 } $\begin{array}{c}\text { Third-order Taylor } \\
\text { approximated range model }\end{array}$ & Range & $0.00 \%$ & -13.26 & -10.04 \\
\cline { 2 - 5 } & Azimuth & $1.35 \%$ & -12.91 & -9.25 \\
\hline
\end{tabular}

\subsection{Validation of the Proposed Imaging Method}

In this subsection, numerical experiments are conducted to investigate the performance of the proposed imaging method. The parameters of CSSAR are given in Table 1. Five moving targets, named T1, T2, T3, T4, and T5, are simulated, and their parameters are presented in Table 3. The signal-to-noise ratio (SNR) is set to be $-10 \mathrm{~dB}$.

Table 3. Parameters of the moving targets.

\begin{tabular}{ccccccc}
\hline & $v_{x}(\mathbf{m} / \mathbf{s})$ & $v_{y}(\mathbf{m} / \mathbf{s})$ & $a_{x}\left(\mathbf{m} / \mathbf{s}^{\mathbf{2}}\right)$ & $a_{y}\left(\mathbf{m} / \mathbf{s}^{\mathbf{2}}\right)$ & $\boldsymbol{r}_{\mathbf{0}}(\mathbf{k m})$ & $\theta_{0}(\mathbf{r a d})$ \\
\hline T1 & -29 & 20 & -0.5 & 0.3 & 15.8 & 0 \\
\hline T2 & -5 & 10 & 0.1 & 0.2 & 15.9 & 0.01 \\
\hline T3 & 21 & 10 & -0.4 & -0.3 & 16.0 & 0 \\
\hline T4 & 5 & -5 & 0.2 & -0.5 & 16.1 & -0.01 \\
\hline T5 & -24 & -20 & 0.5 & 0.4 & 16.2 & 0 \\
\hline
\end{tabular}




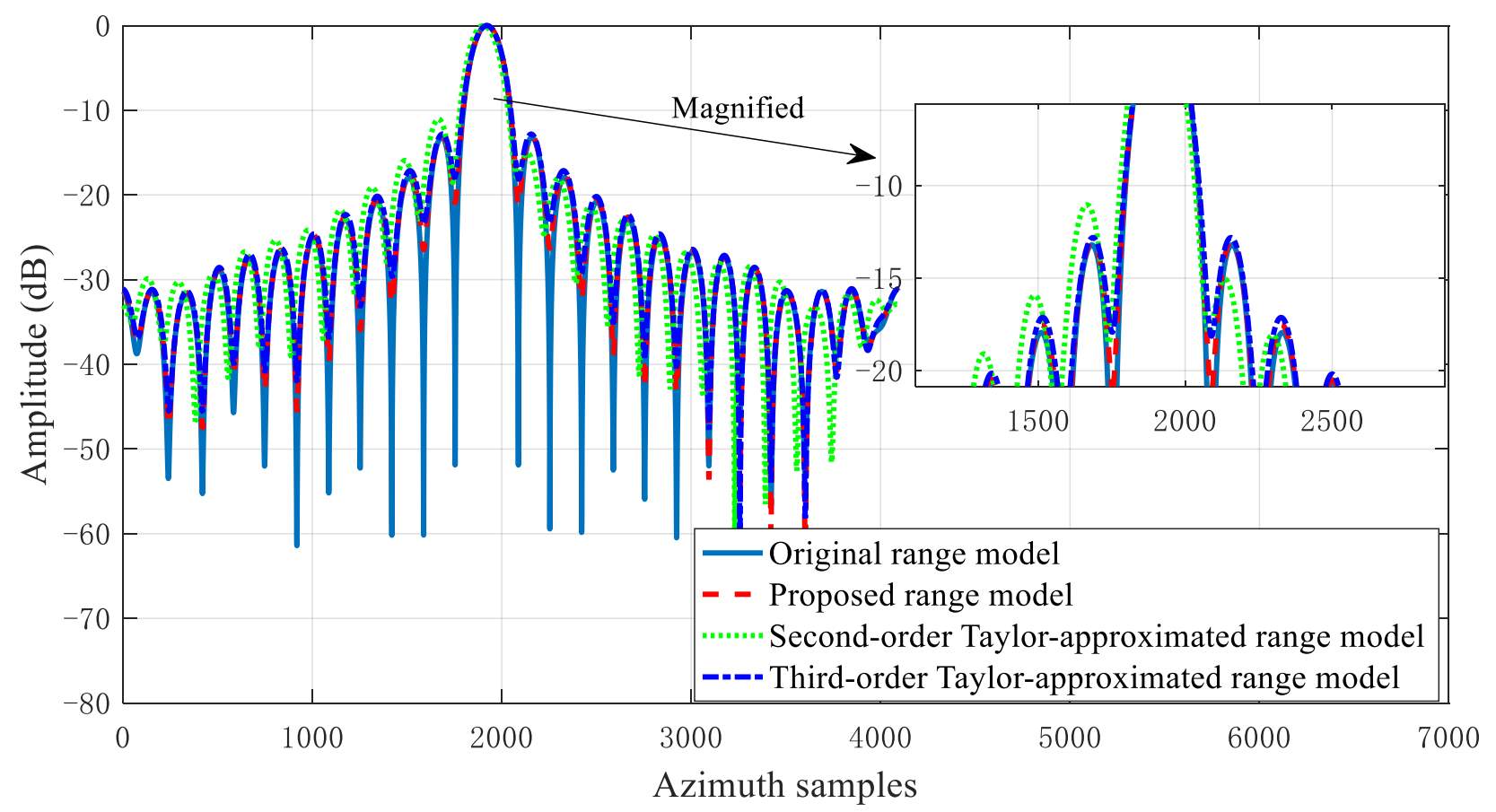

(a)

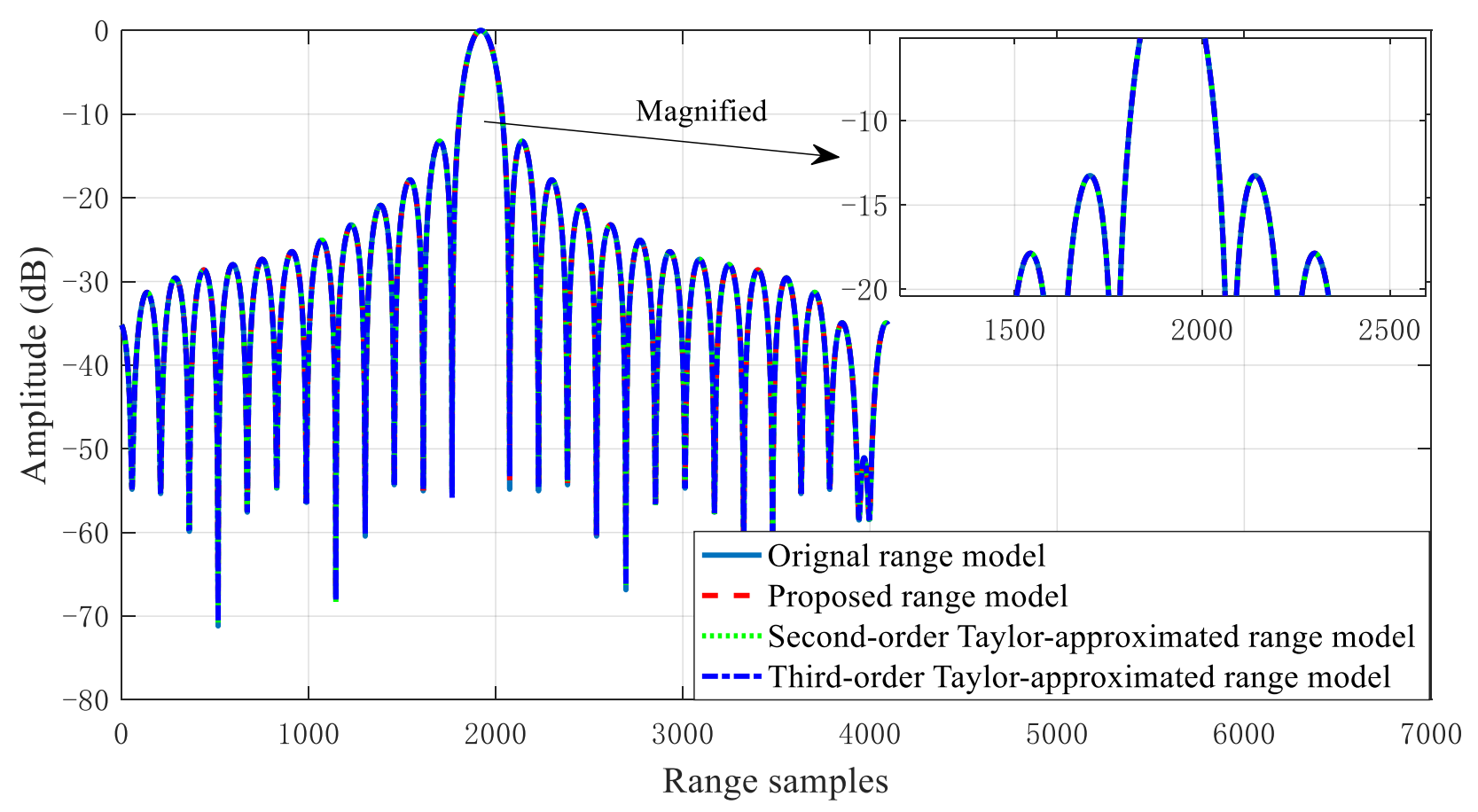

(b)

Figure 5. Focusing results. (a) azimuth profile, (b) range profile. The parameters of the target are $R_{c}=21 \mathrm{~km}, v_{x}=3 \mathrm{~m} / \mathrm{s}, v_{y}=-12 \mathrm{~m} / \mathrm{s}, a_{x}=0.6 \mathrm{~m} / \mathrm{s}^{2}$ and $a_{y}=-0.3 \mathrm{~m} / \mathrm{s}^{2}$.

In using the GA, first, the value ranges of the parameters to be searched and the encoding accuracies should be determined so that we can encode these parameters. In this paper, it is assumed that $v_{x} \in[-30 \mathrm{~m} / \mathrm{s}, 30 \mathrm{~m} / \mathrm{s}], v_{y} \in[-30 \mathrm{~m} / \mathrm{s}, 30 \mathrm{~m} / \mathrm{s}], a_{x} \in\left[-1 \mathrm{~m} / \mathrm{s}^{2}, 1 \mathrm{~m} / \mathrm{s}^{2}\right]$, $a_{y} \in\left[-1 \mathrm{~m} / \mathrm{s}^{2}, 1 \mathrm{~m} / \mathrm{s}^{2}\right]$. Then, according to (3)-(9) and (12)-(14), the value ranges of $v_{e}, \alpha$, and $\beta$ can be figured out and the results are: $v_{e} \in[-297.31 \mathrm{~m} / \mathrm{s}, 362.59 \mathrm{~m} / \mathrm{s}], \alpha \in\left[-7.75 \times 10^{5} \mathrm{~m}^{2} / \mathrm{s}\right.$, 
$\left.7.75 \times 10^{5} \mathrm{~m}^{2} / \mathrm{s}\right], \beta \in[-50.34 \mathrm{~m} / \mathrm{s}, 50.34 \mathrm{~m} / \mathrm{s}]$. The encoding accuracies for the three parameters can be set according to the required focusing quality. To keep the azimuth impulse response width (IRW) broadening within $2 \%$, the Doppler chirp rate error should be less than $1 / T_{a}^{2}$, where $T_{a}$ is the synthetic aperture time [16]. The Doppler chirp rate is related to $v_{e}$ and $\alpha$, and their relationship is as follows

$$
K_{a}=-\frac{4}{\lambda}\left(\frac{v_{e}^{2}}{2 R_{c}}-\frac{\alpha^{2}}{8 R_{c}^{3}}\right)
$$

In the experiments, the bound for the Doppler chirp rate error is set to be $1 /\left(3 T_{a}^{2}\right)$. Then, based on (30), the encoding accuracies for $v_{e}$ and $\alpha$ can be given by (31) and (32), respectively.

$$
\begin{gathered}
\Delta v_{e}=\min _{v_{e}}\left\{-v_{e}+\sqrt{v_{e}^{2}+\frac{\lambda R_{c}}{12 T_{a}^{2}}}\right\} \\
\Delta \alpha=\min _{\alpha}\left\{-\alpha+\sqrt{\alpha^{2}+\frac{\lambda R_{c}^{3}}{3 T_{a}^{2}}}\right\}
\end{gathered}
$$

The derivation for (31) and (32) are presented in Appendix A. As for $\beta$, the Doppler center frequency is depended on it and their relationship is formulated in (33). Based on the imaging method shown in (27), the estimation accuracy of $\beta$ should keep us obtain an accurate Doppler ambiguity. In the simulations, the encoding accuracy for $\beta$ is given by (34), which could make the estimation error for the Doppler center frequency be less than $\mathrm{PRF} / 10$, where PRF is the pulse repetition frequency.

$$
\begin{gathered}
f_{a c}=-\frac{2}{\lambda}\left(\beta+\frac{\alpha}{2 R_{c}}\right) \\
\Delta \beta=\lambda \cdot \frac{P R F}{20}
\end{gathered}
$$

According to (31), (32), and (34), the encoding accuracies for the three parameters are figured out, and the results are: $\Delta v_{e}=0.03 \mathrm{~m} / \mathrm{s}, \Delta \alpha=9000 \mathrm{~m}^{2} / \mathrm{s}, \Delta \beta=2.25 \mathrm{~m} / \mathrm{s}$. In addition, the population size is set to be 50 , and the maximum generation is set to be 40 . After these parameters being set, the strategy presented in Section 4.2 is utilized to solve the imaging optimization problem. The execution time for each target is measured, which shows that it needs only about $24 \mathrm{~min}$ to obtain the optimal solution. The solving processes for the five targets are illustrated in Figure 6, with the optimal image contrasts of each generation being plotted. As can be seen from Figure 6, the proposed method converges quickly.

Figure 7 shows the imaging results, with the contours of target energy being plotted. The results are obtained by extracting a window of $64 \times 64$ samples centered on the target and then expanding this "window" by a factor of 64 via zero padding the spectrum and inverse transforming. In addition, the image quality parameters are measured and the results are shown in Table 4 . The ideal ISLR is measured from the ideal sinc function with the same bandwidth. From Figure 7 it can be seen that the five targets are all finely focused. Moreover, from Table 4 one can see that the image quality parameters are all very close to their theoretical values, which indicates that the targets are accurately focused by the proposed imaging method. 


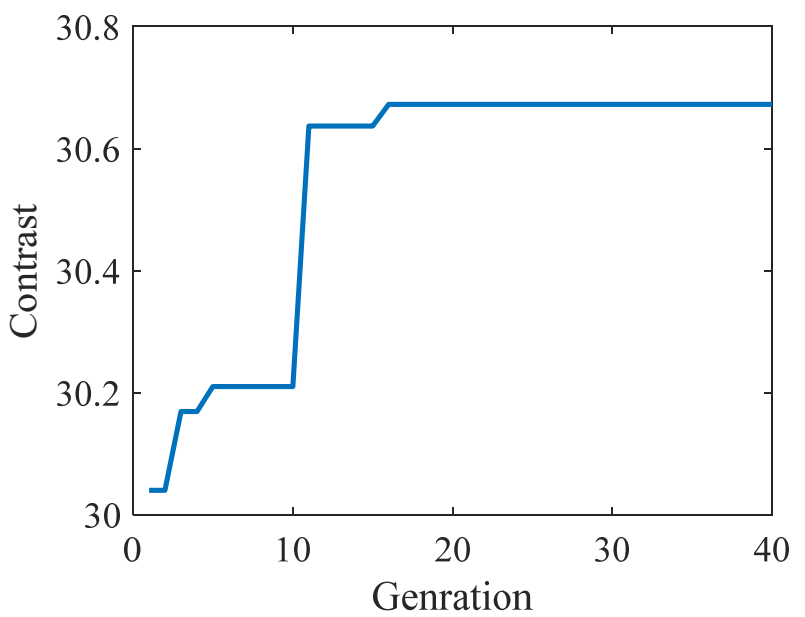

(a)

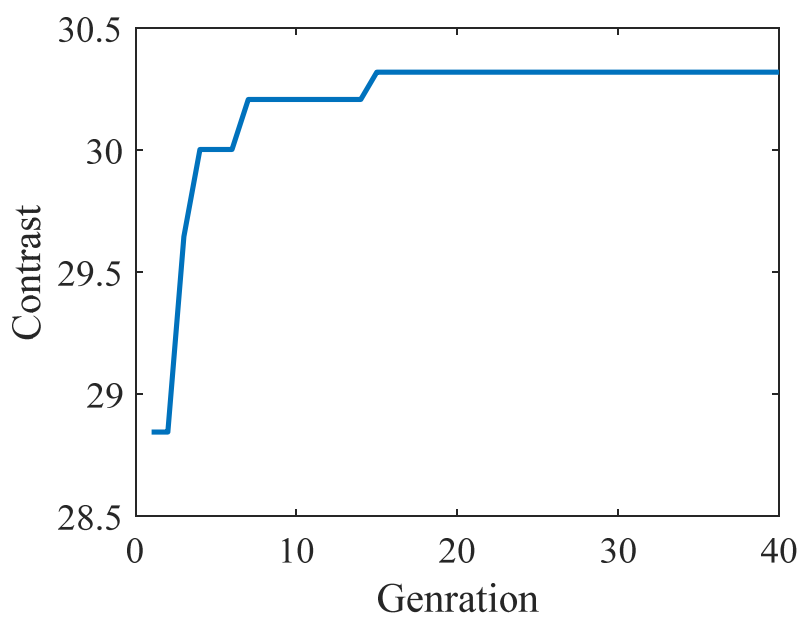

(c)

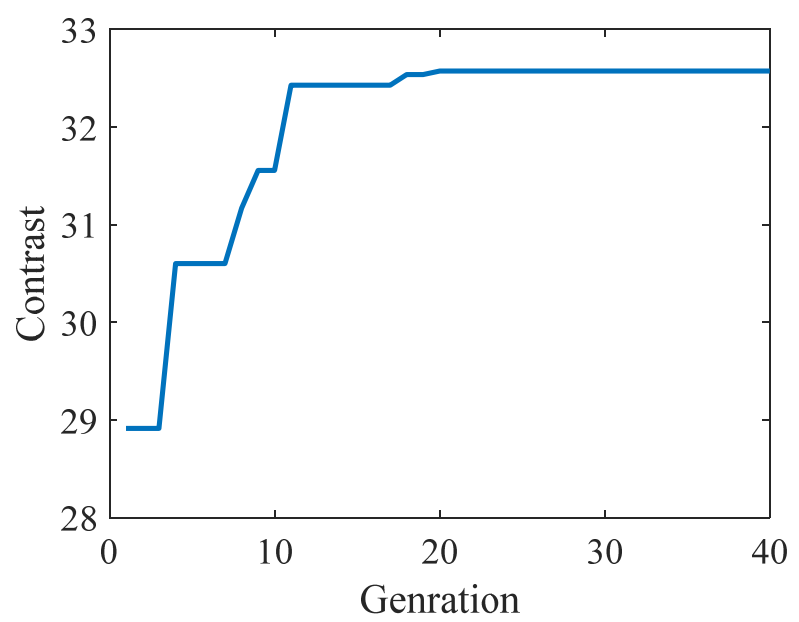

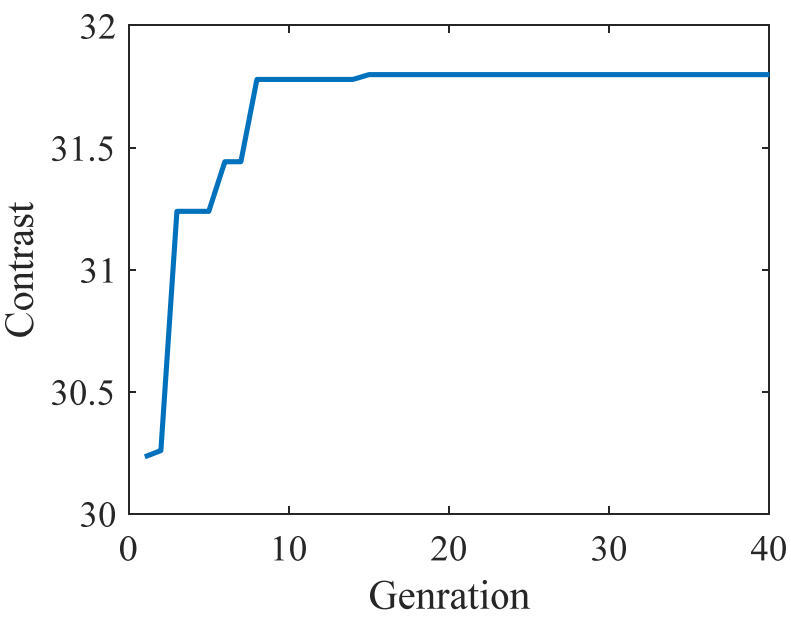

(b)

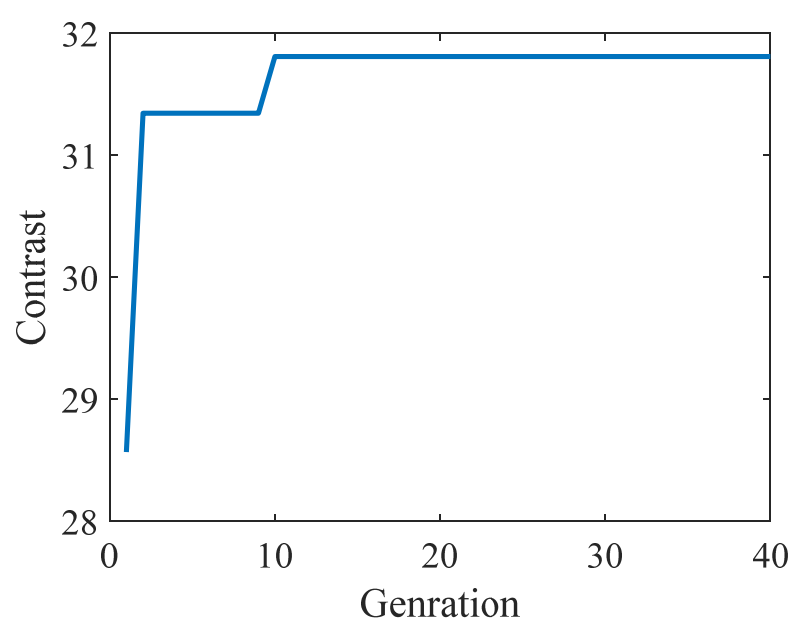

(d)

(e)

Figure 6. Solving processes of the optimization problem. (a) T1, (b) T2, (c) T3, (d) T4, and (e) T5. 


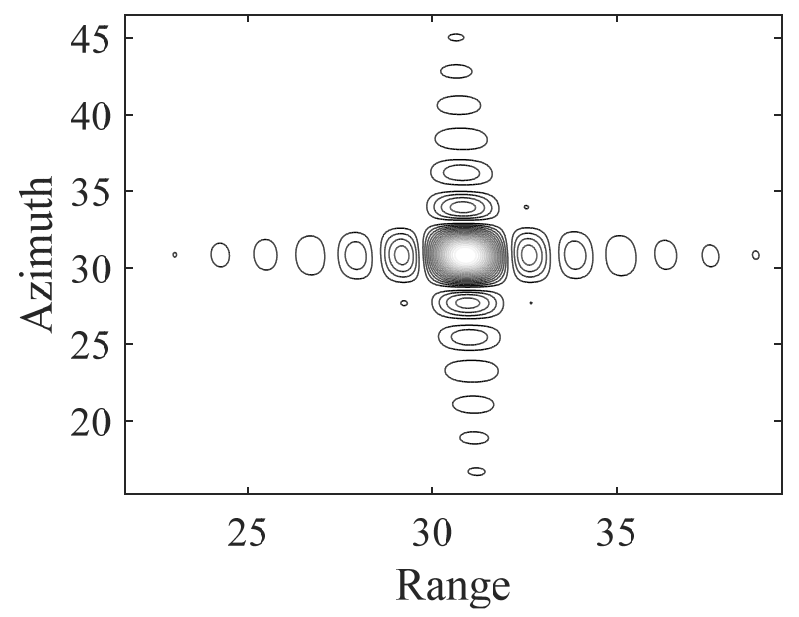

(a)

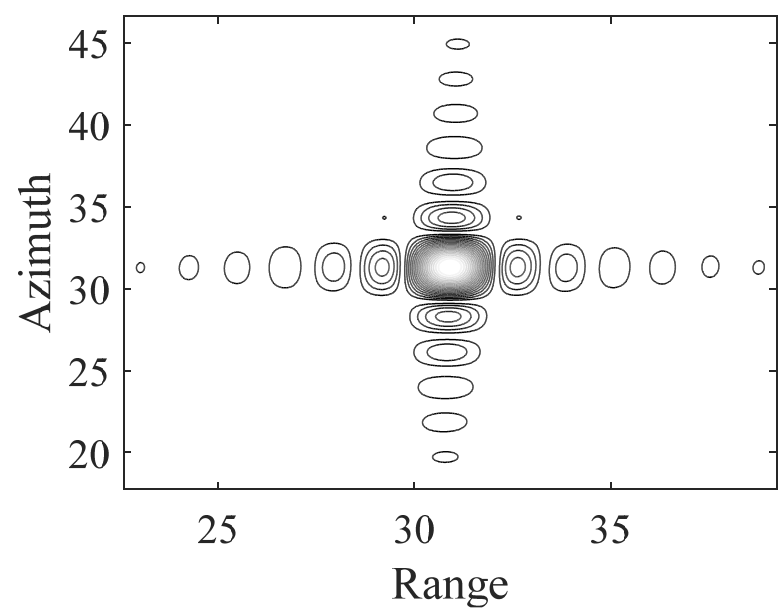

(c)

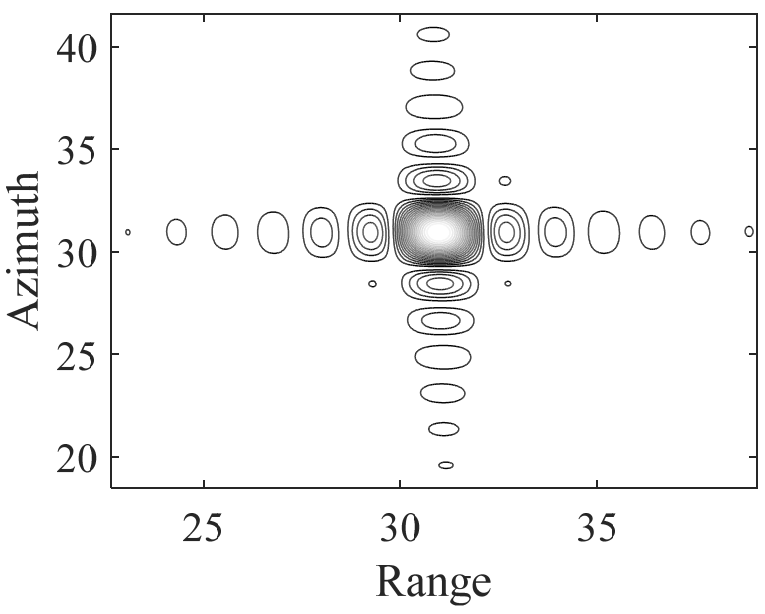

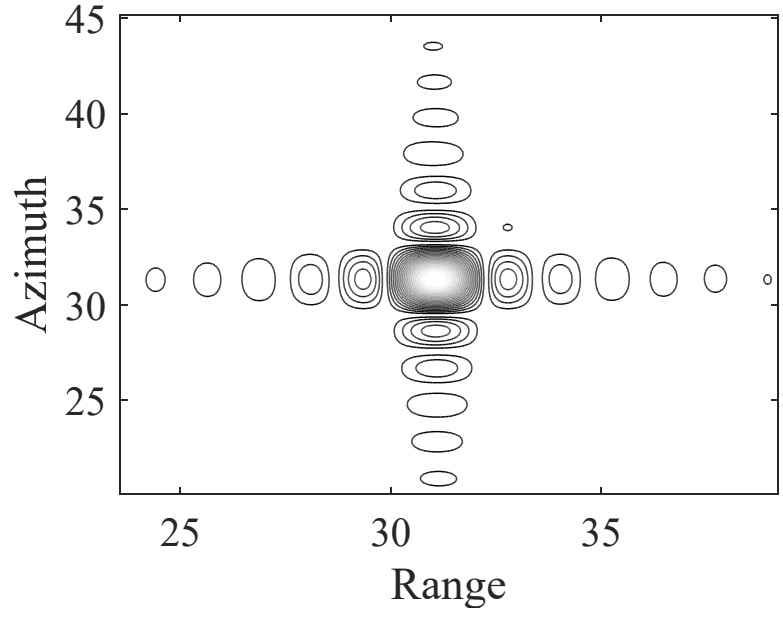

(b)

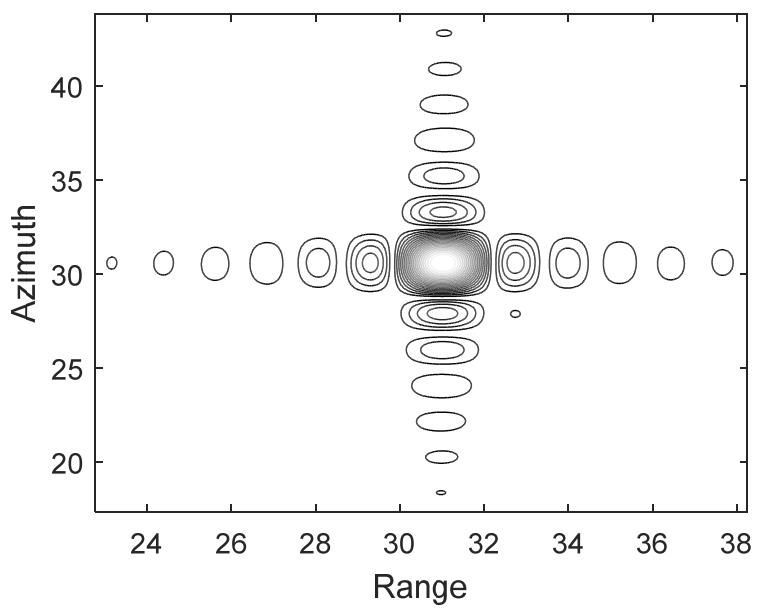

(d)

(e)

Figure 7. Contour plots of the five targets. (a) T1, (b) T2, (c) T3, (d) T4, and (e) T5. 
Table 4. Measured image quality parameters.

\begin{tabular}{cccccc}
\hline & & IRW Broadening & $\begin{array}{c}\text { PSLR } \\
\text { (dB) }\end{array}$ & $\begin{array}{c}\text { ISLR } \\
\text { (dB) }\end{array}$ & $\begin{array}{c}\text { Ideal ISLR } \\
\text { (dB) }\end{array}$ \\
\hline \multirow{2}{*}{$\mathrm{T} 1$} & Azimuth & $0.81 \%$ & -13.21 & -10.02 & -10.01 \\
\cline { 2 - 6 } & Range & $0.00 \%$ & -13.16 & -9.79 & -9.86 \\
\hline \multirow{2}{*}{$\mathrm{T} 2$} & Azimuth & $0.00 \%$ & -13.22 & -9.90 & -9.96 \\
\cline { 2 - 6 } & Range & $0.00 \%$ & -13.22 & -9.80 & -9.86 \\
\hline \multirow{2}{*}{$\mathrm{T} 3$} & Azimuth & $0.00 \%$ & -13.26 & -9.98 & -10.00 \\
\cline { 2 - 6 } & Range & $0.00 \%$ & -13.27 & -9.88 & -9.86 \\
\hline \multirow{2}{*}{$\mathrm{T} 4$} & Azimuth & $0.00 \%$ & -13.25 & -9.95 & -9.96 \\
\cline { 2 - 6 } & Range & $0.00 \%$ & -13.22 & -9.80 & -9.86 \\
\hline \multirow{2}{*}{$\mathrm{T} 5$} & Azimuth & $0.00 \%$ & -13.18 & -9.89 & -9.94 \\
\cline { 2 - 6 } & Range & $0.00 \%$ & -13.27 & -9.90 & -9.86 \\
\hline
\end{tabular}

\section{Discussions}

The experimental results presented in Section 5 validate the proposed range model and the proposed imaging method. In Section 5.1, the qualities of the results obtained based on the original range equation, the proposed range model, the second-order and third-order Taylor-approximated range models are measured and compared. The results demonstrate that the accuracy of the proposed range model is better than that of the second-order and third-order Taylor-approximated range models, and that one can obtain a high-quality focusing result with the proposed range model. In Section 5.2, the imaging results obtained by the proposed method are analyzed. It is seen that the image qualities of the five targets are all close to the theoretical values. Therefore, the proposed imaging method can focus ground moving targets accurately.

The computational load of the proposed imaging method is discussed as follows. According to the analyzes presented in Section 4, the major operations of the proposed method include twice 2-D Fourier transform, twice inverse 2-D Fourier transform, and a 2-D phase multiplication. Assuming that the number of azimuth samples and range samples are $N_{a}$ and $N_{r}$, respectively. Then, the total number of floating operations can be calculated as:

$$
20 N_{r} N_{a} \log _{2}\left(N_{r}\right)+20 N_{r} N_{a} \log _{2}\left(N_{a}\right)+6 N_{r} N_{a}
$$

Therefore, the computational order of the proposed method is $O\left(N^{2} \log _{2} N\right)$, where $N$ denotes the 1-D size of the data.

GA is a parallel stochastic algorithm, and it may not converge to the optimal result in a few cases. Now we discuss the variability and statistical significance of the results obtained by GA. Five hundred random experiments are conducted, with the target T1 being considered. The results obtained by these random experiments are analyzed, and the deviations of the obtained optimal image contrasts are plotted in Figure 8. It is found out that there are 479 out of 500 experiments converge to the optimal results. Moreover, it is also figured out that the maximum deviation, the mean deviation, and the standard deviation of the results are $0.1007,0.0034$ and 0.0178 , respectively, which are all very small. 


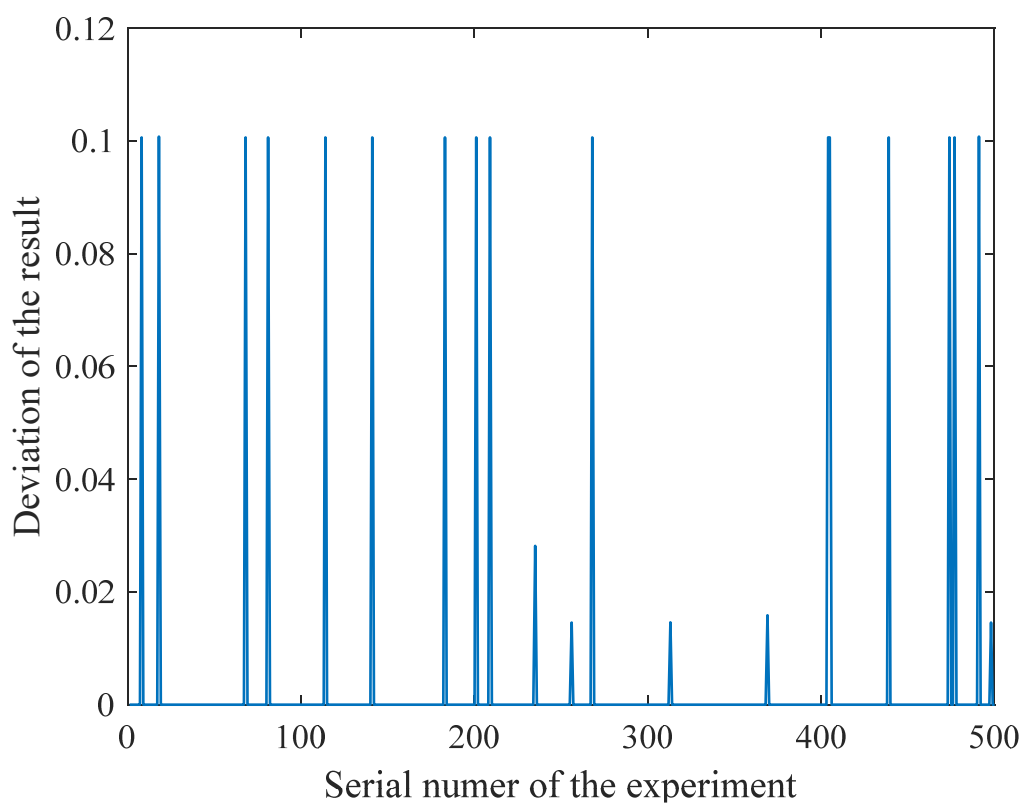

Figure 8. Deviations of the optimal image contrasts obtained by 500 random experiments.

\section{Conclusions}

In this paper, an accurate range model and an efficient ground moving target imaging method have been proposed for airborne CSSAR. The main merits of the proposed range model are highlighted in two aspects: (1) its accuracy is even higher than that of the thirdorder Taylor approximated range equation; and (2) based on the proposed range model, the accurate analytical expression for the target's 2-D spectrum can be easily derived. Moreover, an efficient ground moving target imaging method has been proposed. The proposed method focuses the target via a phase multiplication in the 2-D frequency domain and utilizes the GA to accomplish an efficient search of the parameters of the proposed range model. Experimental results have validated the proposed imaging method.

Author Contributions: The work presented in this manuscript was accomplished together with all of the authors. Y.L. proposed the idea, carried out the theoretical derivations and analyses, conducted the experiments, and wrote the manuscript. T.H. conducted the theoretical derivations, carried out numerical simulations, and reviewed the manuscript. T.W. validated the proposed method and reviewed the manuscript. J.W. managed the project and reviewed the manuscript. C.Y. conducted the simulations and reviewed the manuscript. B.L. reviewed the manuscript and wrote some codes. All authors have read and agreed to the published version of the manuscript.

Funding: This paper was funded by the Postdoctoral Science Foundation of China under Grants BX20180261 and 2019M653739, by the Aeronautical Science Foundation of China under Grant ASFC20200020053001, by the National Natural Science Foundation of China under Grant 61701413, by the Undergraduate Innovation and Entrepreneurship Training Program under Grant S202010699318, by the Natural Science Foundation of Shaanxi Province under Grant 2018JQ6095, and by the Natural Science Basic Research Plan in Shaanxi Province of China under Grant 2020JQ-203.

Institutional Review Board Statement: Not applicable.

Informed Consent Statement: Not applicable.

Data Availability Statement: The data presented in this study are available on request from the corresponding author.

Acknowledgments: The authors would like to thank the Wang, L. for his help in preparing this paper. They would also like to thank the reviewers for their valuable comments, and Cao, J. W., Bi G. A. and $\mathrm{Wu}, \mathrm{X}$. T. for proofreading the manuscript.

Conflicts of Interest: The authors declare no conflict of interest. 


\section{Symbols}

The symbols used in this paper are summarized as follows

$h$
Height of the aircraft

Angular velocity of the aircraft

Flying radius of the aircraft

Azimuth slow time

Distance from the target to the coordinate origin

Azimuth angle of the target

Target's velocity along the $x$-axis

Target's velocity along the $y$-axis

Target's acceleration along the $x$-axis

Target's acceleration along the y-axis

Distance from the target to the radar at the beam center crossing time

Beam center crossing time of the target

Coefficient of the first-order term of the Taylor-approximated range model

Coefficient of the second-order term of the Taylor-approximated range model

Coefficient of the third-order term of the Taylor-approximated range model

Distance from the target to the coordinate origin at the beam center crossing time Projection of the target's velocity onto the vertical direction of the radar platform velocity at the beam center crossing time

Projection of the target's acceleration onto the vertical direction of the radar platform velocity at the beam center crossing time

Projection of the target's velocity onto the direction of the radar platform velocity at the beam center crossing time

Projection of the target's acceleration onto the direction of the radar platform velocity at the beam center crossing time

Target's azimuth angle at the beam center crossing time

Variable introduced in the proposed range model, its unit is $\mathrm{m} / \mathrm{s}$

Variable introduced in the proposed range model, its unit is $\mathrm{m}^{2} / \mathrm{s}$

Variable introduced in the proposed range model, its unit is $\mathrm{m} / \mathrm{s}$

Synthetic aperture time

$3 \mathrm{db}$ beamwidth of the radar

Azimuth resolution

Velocity of the beam footprint along the ground

Velocity of the radar platform

Range envelope

Azimuth envelope

Range time

Chirp rate of the transmitted signal

Speed of light

Carrier frequency

Range frequency

Range frequency envelope

Azimuth frequency

Azimuth frequency envelope

Length of the string for $v_{e}$

Encoding accuracy for $v_{e}$

nth chromosome of the population in gth generation

Doppler chirp rate

Encoding accuracy for $\alpha$

Encoding accuracy for $\beta$

Pulse repetition frequency

Doppler center frequency

Number of azimuth samples of data

Number of range samples of data

1-D size of the data 


\section{Appendix A}

This appendix drives the encoding accuracies for $v_{e}$ and $\alpha$.

First, the derivation of the encoding accuracy for $v_{e}$ is presented. Since the bound for the Doppler chirp rate error is set to be $1 /\left(3 T_{a}^{2}\right)$, according to the relationship between the Doppler chirp rate and $v_{e}$ shown in (30), the encoding accuracy for $v_{e}$ (i.e., $\Delta v_{e}$ ) should satisfy the following equation

$$
\frac{1}{6 T_{a}^{2}}=\frac{4}{\lambda} \cdot \frac{\left(v_{e}+\Delta v_{e}\right)^{2}-v_{e}^{2}}{2 R_{c}}
$$

By solving the above quadratic equation of one unknown, the solution for $\Delta v_{e}$ can be expressed as

$$
\Delta v_{e}=-v_{e}+\sqrt{v_{e}^{2}+\frac{\lambda R_{c}}{12 T_{a}^{2}}}
$$

Since $v_{e}$ ranges from $v_{e, \min }$ to $v_{e, \text { max }}$, the encoding accuracy for $v_{e}$ can be calculated by

$$
\Delta v_{e}=\min _{v_{e}}\left\{-v_{e}+\sqrt{v_{e}^{2}+\frac{\lambda R_{c}}{12 T_{a}^{2}}}\right\}
$$

Now, we present the derivation of the encoding accuracy for $\alpha$. Similar to the derivation of $\Delta v_{e}$, according to (30), the encoding accuracy for $\alpha$ (i.e., $\Delta \alpha$ ) should satisfy the following equation

$$
\frac{1}{6 T_{a}^{2}}=\frac{4}{\lambda} \cdot \frac{(\alpha+\Delta \alpha)^{2}-\alpha^{2}}{8 R_{c}^{3}}
$$

The solution for the above quadratic equation of one unknown is given by

$$
\Delta \alpha=-\alpha+\sqrt{\alpha^{2}+\frac{\lambda R_{c}^{3}}{3 T_{a}^{2}}}
$$

Since $\alpha$ ranges from $\alpha_{\min }$ to $\alpha_{\max }$, the encoding accuracy for $\alpha$ can be calculated by

$$
\Delta \alpha=\min _{\alpha}\left\{-\alpha+\sqrt{\alpha^{2}+\frac{\lambda R_{c}^{3}}{3 T_{a}^{2}}}\right\}
$$

\section{References}

1. Moreira, A.; Prats-iraola, P.; Younis, M.; Krieger, G.; Hajnsek, I.P.; Papathanassiou, K. A Tutorial on Synthetic Aperture Radar. IEEE Mag. Geosci. Remote Sens. 2013, 1, 6-43. [CrossRef]

2. Jäger, M.; Pinheiro, M.; Ponce, O.; Reigber, A.; Scheiber, R. A Survey of Novel Airborne SAR Signal Processing Techniques and Applications for DLR's F-SAR Sensor. In Proceedings of the 16th International Radar Symposium (IRS), Dresden, Germany, 24-26 June 2015; pp. 1-6.

3. Cantalloube, H.M.J.; Nahum, C.E. Airborne SAR-Efficient Signal Processing for Very High Resolution. Proc. IEEE. 2013, 3, 784-797. [CrossRef]

4. $\quad$ Reigber, A.; Scheiber, R.; Jager, M.; Prats-Iraola, P.; Hajnsek, I.; Jagdhuber, T.; Papathanassiou, K.P.; Nannini, M.; Aguilera, E.; Baumgartner, S.; et al. Very-High-Resolution Airborne Synthetic Aperture Radar Imaging: Signal Processing and Applications. Proc. IEEE 2013, 3, 759-783. [CrossRef]

5. Ren, Y.; Tang, S.Y.; Guo, P.; So, H.C.; Zhang, L.R. 2-D Spatially Variant Motion Error Compensation for High-Resolution Airborne SAR Based on Range-Doppler Expansion Approach. IEEE Trans. Geosci. Remote Sens. 2021, in press. [CrossRef]

6. Jao, J.K. Theory of Synthetic Aperture Radar Imaging of a Moving Target. IEEE Trans. Geosci. Remote Sens. 2001, 9, $1984-1992$.

7. Bacci, A.; Martorella, M.; Gray, D.A.; Gelli, S.; Berizzi, F. Virtual Multichannel SAR for Ground Moving Target Imaging. IET Radar Sonar Navigat. 2016, 1, 50-62. [CrossRef]

8. Zhu, D.Y.; Li, Y.; Zhu, Z.D. A Keystone Transform Without Interpolation for SAR Ground Moving-Target Imaging. IEEE Geosci. Remote Sens. Lett. 2007, 1, 18-22. [CrossRef] 
9. Wan, J.; Tan, X.; Chen, Z.; Dong, L.; Liu, Q.; Zhou, Y.; Zhang, L. Refocusing of Ground Moving Targets with Doppler Ambiguity Using Keystone Transform and Modified Second-Order Keystone Transform for Synthetic Aperture Radar. Remote Sens. 2021, 2, 177. [CrossRef]

10. Zhu, S.Q.; Liao, G.S.; Qu, Y.; Zhou, Z.G.; Liu, X.Y. Ground Moving Targets Imaging Algorithm for Synthetic Aperture Radar. IEEE Trans. Geosci. Remote Sens. 2011, 1, 462-477. [CrossRef]

11. Baumgartner, S.V.; Krieger, G. Dual-Platform Large Along-Track Baseline GMTI. IEEE Trans. Geosci. Remote Sens. 2016, 3, 1554-1574. [CrossRef]

12. Hashemi, S.R.S.; Bayat, S.; Nayebi, M.M. Ground-Based Moving Target Imaging in a Circular Strip-Map Synthetic Aperture Radar. In Proceedings of the IEEE 5th Asia-Pacific Conference on Synthetic Aperture Radar (APSAR), Singapore, 1-4 October 2015; pp. 835-840.

13. Shao, Y.; Chen, Z.Y.; Wu, X.B. Investigation on Imaging Performance of Circular Scanning Synthetic Aperture Radar. In Proceedings of the XXXIth URSI General Assembly and Scientific Symposium (URSI GASS), Beijing, China, 16-23 August 2014; pp. 1-4.

14. Liao, Y.; Wang, W.Q.; Liu, Q.H. Two-Dimensional Spectrum for Circular Trace Scanning SAR Based on an Implicit Function. IEEE Geosci. Remote Sens. Lett. 2016, 7, 887-891. [CrossRef]

15. Baumgartner, S.V. Circular and Polarimetric ISAR Imaging of Ships Using Airborne SAR Sensors. In Proceedings of the 12th European Conference on Synthetic Aperture Radar (EUSAR), Berlin, Germany, 4-7 June 2018; pp. 1-6.

16. Cumming, I.G.; Wong, F.H. Digital Processing of Synthetic Aperture Radar Data: Algorithm and Implementation; Artech House: Norwood, MA, USA, 2004.

17. Sun, G.C.; Xing, M.D.; Xia, X.G.; Wu, Y.R.; Bao, Z. Robust Ground Moving-Target Imaging Using Deramp-Keystone Processing IEEE Trans. Geosci. Remote Sens. 2013, 2, 966-982. [CrossRef]

18. Wang, G.Y.; Xia, X.G.; Chen, V.C. Dual-Speed SAR Imaging of Moving Targets. IEEE Trans. Aerosp. Electron. Syst. 2006, 1, 368-379. [CrossRef]

19. Noviello, C.; Fornaro, G.; Martorella, M. Focused SAR Image Formation of Moving Targets Based on Doppler Parameter Estimation. IEEE Trans. Geosci. Remote Sens. 2015, 2, 966-982. [CrossRef]

20. Baumgartner, S.V.; Krieger, G. Multi-Channel SAR for ground Moving Target Indication. Elsevier Acad. Press Libr. Signal Process. 2014, 2, 911-986.

21. Zhang, X.P.; Liao, G.S.; Zhu, S.Q.; Zeng, C.; Shu, Y.X. Geometry-Information-Aided Efficient Radial Velocity Estimation for Moving Target Imaging and Location Based on Radon Transform. IEEE Trans. Geosci. Remote Sens. 2015, 2, 1105-1117. [CrossRef]

22. Huang, P.H.; Liao, G.S.; Yang, Z.; Xia, X.G.; Ma, J.T.; Zheng, J.B. Ground Maneuvering Target Imaging and High-Order Motion Parameter Estimation Based on Second-Order Keystone and Generalized Hough-HAF Transform. IEEE Trans. Geosci. Remote Sens. 2017, 1, 320-335. [CrossRef]

23. Zeng, C.; Li, D.; Luo, X.; Liu, H.Q.; Su, J. Ground Maneuvering Targets Imaging for Synthetic Aperture Radar Based on SecondOrder Keystone Transform and High-Order Motion Parameter Estimation. IEEE J. Sel. Topics Appl. Earth Observ. Remote Sens. 2019, 11, 4486-4501. [CrossRef]

24. Li, D.; Ma, H.N.; Liu, H.Q.; Chen, Z.Y.; Su, J.; Zhou, X.C.; Li, W.; Yang, Z.J. An Efficient Ground Maneuvering Target Refocusing Method Based on Principal Component Analysis and Motion Parameter Estimation. Remote Sens. 2020, 12, 1-22. [CrossRef]

25. Huang, L.J.; Qiu, X.; Hu, D.; Ding, C. Focusing of Medium-Earth-Orbit SAR with Advanced Nonlinear Chirp Scaling Algorithm. IEEE Trans. Geosci. Remote Sens. 2011, 49, 500-508. [CrossRef]

26. Li, Y.K.; Wang, T.; Liu, B.C.; Hu, R.X. High-Resolution SAR Imaging of Ground Moving Targets Based on the Equivalent Range Equation. IEEE Geosci. Remote Sens. Lett. 2015, 2, 324-328.

27. Zhang, Y.; Sun, J.P.; Lei, P.; Li, G.; Hong, W. High-Resolution SAR-Based Ground Moving Target Imaging With Defocused ROI Data. IEEE Trans. Geosci. Remote Sens. 2016, 2, 1062-1073. [CrossRef]

28. Jin, G.H.; Dong, Z.; He, F.; Yu, A.X. SAR Ground Moving Target Imaging Based on a New Range Model Using a Modified Keystone Transform. IEEE Trans. Geosci. Remote Sens. 2019, 6, 3283-3295. [CrossRef]

29. Jao, J.K.; Yegulalp, A. Multichannel Synthetic Aperture Radar Signatures and Imaging of a Moving Target. Inverse Probl. 2013, 5, 1-33. [CrossRef]

30. Yang, J.; Liu, C.; Wang, Y.F. Imaging and Parameter Estimation of Fast-Moving Targets with Single-Antenna SAR. IEEE Geosci. Remote Sens. Lett. 2014, 2, 529-533. [CrossRef]

31. Yang, L.; Zhao, L.F.; Bi, G.A.; Zhang, L.R. SAR Ground Moving Target Imaging Algorithm Based on Parametric and Dynamic Sparse Bayesian Learning. IEEE Trans. Geosci. Remote Sens. 2016, 4, 2254-2267. [CrossRef]

32. Srinivas, M.; Patnaik, L.M. Genetic Algorithms: A Survey. Computer 1994, 6, 17-26. [CrossRef]

33. Whitley, D. A Genetic Algorithm Tutorial. Stattics Comput. 1994, 4, 65-85. [CrossRef]

34. Zhang, J.; Chung, H.S.H.; Lo, W.L. Clustering-Based Adaptive Crossover and Mutation Probabilities for Genetic Algorithms. IEEE Trans. Evol. Comput. 2007, 3, 326-335. [CrossRef]

35. Chen, Y.J.; Zhang, Q.; Luo, Y.; Chen, Y.A. Measurement Matrix Optimization for ISAR Sparse Imaging Based on Genetic Algorithm. IEEE Trans. Geosci. Remote Sens. 2016, 12, 1875-1879. [CrossRef] 
36. Shi, L.; Deng, Y.K.; Sun, H.F.; Wang, R.; Ai, J.Q.; Yan, H. An Improved Real-Coded Genetic Algorithm for the Beam Forming of Spaceborne SAR. IEEE Trans. Antennas Propag. 2012, 6, 3034-3040. [CrossRef]

37. Zhang, T.; Liao, G.S.; Li, Y.C.; Gu, T.; Zhang, T.H.; Liu, Y.J. A Two-Stage Time-Domain Autofocus Method Based on Generalized Sharpness Metrics and AFBP. IEEE Trans. Geosci. Remote Sens. 2021, in press. [CrossRef] 